\title{
Darwinism Without Selection? A Lesson from Cultural Evolutionary Theory
}

\section{LorenzoBaravalle 1\Email 1baravalle@fc.ul.pt}

1 Centro de Filosofia das Ciências, Departamento de História e Filosofia das Ciências, Faculdade de Ciências, Universidade de Lisboa Lisbon Portugal

\section{Abstract}

Wondering about "how Darwinian" cultural change actually is, some authors have recently stressed that there are different degrees to which a process can be considered as evolutionary. Some of them advocate for a central role of selective processes in cultural evolution, while others deny that these are relevant to explain cultural change, if not incidentally. Taking a cue from this debate, in this chapter, I shall discuss a series of theoretical and explanatory commitments usually adopted by those that, like cultural evolutionists, aim to extend evolutionary theory to non-strictly biological domains. My goal is to identify a class of evolutionary factors that, although frequently neglected in the debate, may be actually qualified as Darwinian and, consequently, argue for a more complete picture of evolutionary change. These factors are demographic factors, that is, factors related to the size, density and structure of populations. After having described in some detail in which way they differ from other causes of evolution, I shall relate the discussion in cultural evolutionary theory to a broader debate about the importance of natural selection in Darwinian thinking.

\section{Keywords}

Cultural evolutionary theory

Cultural change

Natural selection

Population thinking

Demographic factors

\section{1 .}

\section{Introduction}

Practitioners in cultural evolutionary theory usually consider themselves as full-fledged Darwinians (e.g. Richerson and Boyd 2005; Mesoudi 2011; Henrich 2016). Nonetheless, wondering about "how Darwinian" cultural change actually is, Peter Godfrey-Smith (2007, 2009), Tim Lewens $(2009,2013,2015)$ and Claidière et al. (2014) have recently stressed that 
there are different degrees to which a process can be considered Darwinian. Beyond the taxonomic and terminological idiosyncrasies of each author (Brusse 2017), all of them agree that there are three main possible Darwinist interpretations of cultural change. They are-from the strictest (i.e. cultural change is taken to be very similar to biological evolution) to the loosest (i.e. cultural change is only vaguely resembling biological evolution)the replicator interpretation, the selectionist interpretation and the populational one. According to the replicator interpretation, cultural change is Darwinian insofar as it involves the propagation of discrete units of information analogous to genes, i.e. the memes (Dawkins 1976; Dennett 1995, 2017; Blackmore 1999). More modestly, the selectionist interpretation assumes that cultures are composed by heterogeneous cultural traits or variants (Boyd and Richerson 1985; Richerson and Boyd 2005) that is, traditions, skills, beliefs, techniques, knowledge, etc.-which not necessarily are similar to genes. Yet, given that (I) there is variation between cultural variants, (II) they are relatively faithfully transmitted and (III) they are differentially adopted in virtue of some of their features, then-following a widely accepted conception (Lewontin 1970; Huneman 2015) - it is still possible to say that cultural change instantiates a Darwinian process. Finally, the populational interpretation denies that these conditions are met-if not occasionally-by cultural change and maintains, instead, that cultural evolutionary theory is "evolutionary" just because it relies on a certain extension of population thinking to the cultural domain. For the authors supporting the populational view, it is somehow misleading to consider cultural change processes as the effect of selective pressures.

The question is: to what extent a theory that does not acknowledge a central role to selection may be properly considered as "Darwinian"? This is an interesting question not just in the context of the debate over cultural evolution but, more in general, for evolutionary biology as a whole. In fact, throughout the last 50 years or so, many practitioners in the fields of molecular evolution (Kimura 1968; King and Jukes 1969), palaeontology (Gould 1977) and developmental biology (Pigliucci and Muller 2010) have challenged, to a different extent, the prominence of natural selection in genetic evolutionary processes (for detailed discussions of this process of reconsideration of the role of natural selection in evolutionary biology and related fields, see Delisle's, Granovitch's, Tattersall's and Schwartz' contributions to this volume).

In this chapter, I shall interpret some recent developments within cultural evolutionary theory as an example of how an evolutionary theory may be considered Darwinian, even though it does not put selection in the foreground. To be precise, I consider that cultural evolutionary theory may be understood as a Wrightean theory. This is because, in addition to explain cultural change by adopting a variational view of populations assimilable to 
Darwin's one, it also puts emphasis - like the well-known "Shifting-balance theory" by Sewall Wright $(1948,1982)$ - on structural properties of populations. To this goal, I shall proceed roughly as follows. First, in Sect. 2, I shall discuss, drawing on Lewtonin (1974), the minimal requirements for a theory to be considered as "evolutionary". An evolutionary theory must provide, necessarily, a set of laws of transformation and it must be dynamically sufficient. I shall thus argue that the disagreement between the supporters of the replicator, the selectionist and the populational interpretations is a disagreement about what makes cultural evolutionary theory dynamically sufficient. I shall discuss the differences between these three interpretations in Sect. 3. In Sect. 4, I shall attempt to go beyond these interpretations, by pointing out that both in evolutionary biology and cultural evolutionary theory, practitioners are paying increasingly more attention to the causal import of demographic factors of evolution. I shall finally argue, in Sect. 5, that the causal role of these factors can be incorporated in a broad Darwinian/Wrightian picture of evolution, in which selection, in spite of being an important engine of change, is not necessarily the fundamental one.

\section{2.}

\section{What Is an Evolutionary Theory?}

In the first chapter of his classic book, The Genetic Basis of Evolutionary Change, Richard Lewontin presents those which, in his opinion, are the basic requirements for a theory to be considered an evolutionary theory. They are very general requirements, and they are likely satisfied also by many theories in physics. They should not be intended, thus, as sufficient conditions to identify evolutionary theories in biology. Since they are, nonetheless, necessary conditions for any theory aimed to account for evolutionary changes, they provide a valuable starting point for our discussion. Lewontin states that "... the problem of constructing an evolutionary theory is the problem of constructing a state space that will be dynamically sufficient, and a set of laws of transformation in that state space that will transform all the state variables" $(1974$, p. 8$)$. In other words, the problem of constructing an evolutionary theory is the problem of providing a set of laws - or we may say, more loosely, a set of models - describing the change of the system under study from an instant $t$ to a subsequent instant $t^{\prime}$, and a suitable causal interpretation of the system, such that the change from $t$ to $t^{\prime}$ can be ascribed to the material factors which are responsible for it.

Thus, for instance, in the genetic theory of evolution, we have a set of equations provided by population genetics - which describe different possible transformations in a state space made up of allelic frequencies - that 
correspond to the laws of transformation of the theory. This theory is, moreover, dynamically sufficient because it identifies, in genetic inheritance and in the differential survival and reproduction of the individuals due to environmental circumstances, the factors that are materially responsible for the transformations in the state space. This is to say that, at least in principle, evolutionary biologists may account for the change in allelic frequencies in a specific population by pointing out, with relative precision, the causes of the change. ${ }^{1}$

Cultural evolutionary theory has received some different mathematical formulations, but the models that are considered by most practitioners as foundational for this disciplinary field are those put forward by CavalliSforza and Feldman (1981) and Boyd and Richerson (1985), within the socalled dual-inheritance theory. These models are an intentional extension of population genetics to changing frequencies of cultural traits, or variants. Cavalli-Sforza, Feldman, Boyd and Richerson interpret cultural traits as a class of phenotypical traits transmitted through a channel of inheritance alternative to the genetic one, that is, social learning. The peculiarity of cultural transmission by social learning is that information does not flow mainly vertically (from parents to offspring generation), like in genetic inheritance, but also horizontally (among the members of the same generation), obliquely (from members of the parental generation to members of the offspring generation who are not genetically related to the former) and even from the offspring to the parental generation. Moreover, while the genetic system of inheritance usually determines a fixed number of ancestors for each descendant, social learning allows some individuals to have a variable number of ancestors. Some individuals adopt a cultural trait after having learned it from different and multiple ancestors. Finally, while genetic inheritance is "discontinuous" (in the sense that an allele is either transmitted to the descendant or not), cultural influence comes in degree (that is, a cultural variant can be just partially transmitted).

The change in the distribution of cultural variants in a population from a generation (taken using any arbitrary time interval) to another is, according to this rather standard picture of cultural evolutionary theory, due to both "purely biological" factors (such as selection, migration, drift, etc.- -after all, cultural variants are phenotypic traits and, as such, are subject to all these evolutionary forces) and other "properly cultural" factors. These have been conceptualised in a variety of ways. Boyd and Richerson (1985; see also Richerson and Boyd 2005), for instance, talk about distinct kinds of social learning biases, which would modify the frequencies of cultural variants depending on the fact that some of them are cognitively more attractive (or more easily memorisable) than others, or are already adopted by prestigious members of the population or by some specific group. Some authors (e.g. Durham 1991; Mesoudi 2011) have suggested to characterise the process 
resulting from the repeated and large-scale action of social learning biases as cultural selection. 2 As we shall see in Sect. 3, other authors consider that this conceptualisation of social learning biases is inaccurate (e.g. Sperber and Claidière 2008; Claidière et al. 2014).

To better understand this criticism we must first, however, consider a preliminary issue. Although all the differences between genetic inheritance and cultural transmission, as well as the effects of the factors producing cultural change, can be, at least in principle, properly formalised (see, for instance, besides the already mentioned Cavalli-Sforza and Feldman 1981; Boyd and Richerson 1985, also Henrich 2001; Henrich and Boyd 2002; El Mouden et al. 2014; Aguilar and Akçay 2018), this is not a guarantee that cultural evolutionary theory is dynamically sufficient. It could be the case, in fact, that we are able to determine the laws of transformation of an evolutionary phenomenon without being able to identify what are, in specific circumstances, the material processes responsible for a certain evolutionary

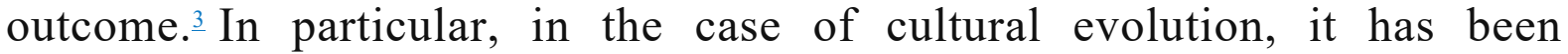
frequently raised the concern that, differently from what occurs with genetic inheritance, we do not have a satisfactory understanding of the causal processes involved in cultural transmission (e.g. Sperber 1996; Aunger 2001).

\section{3.}

\section{Dynamical Sufficiency in Cultural Evolution}

In this section, I shall discuss the three most common interpretations of cultural evolutionary theory as a Darwinian theory. In accordance with the framework presented in the last section, I will argue that they differ mainly because their alternative characterisations of the dynamical features of the processes of cultural change. The replicator interpretation (Sect. 3.1) settles the problem concerning the dynamical sufficiency of cultural evolutionary theory by postulating that the diffusion of cultural traits depends on the cultural transmission of discrete entities analogues to genes, also known as memes. The selectionist interpretation (Sect. 3.2) avoids such a strong analogy between genetic and cultural evolution and grounds the dynamical sufficiency of cultural evolutionary theory on the population-level properties of the processes of cultural change. Finally, the populational interpretation (Sect. 3.3) raises doubts over the correctness of the selectionist interpretation and suggests a deflationary perspective about the dynamical properties of cultural evolutionary processes per se. Cultural change, according to this view, would be epiphenomenal with respect to the myriads of social interactions between the members of a population. In the last Sect. 3.4, I shall draw some partial conclusion about the current status of the debate. 


\subsection{1.}

\section{The Replicator Interpretation}

Originally put forward by Richard Dawkins in his best-seller The Selfish Gene (Dawkins 1976), and later popularised by Dennett (1995) and Blackmore (1999), the so-called memetic theory has, at its core, the thesis that the processes of cultural change are carried out by hypothetical entities, i.e. the memes-which would replicate analogously to genes. The problem of the dynamical sufficiency in cultural evolutionary theory is thus easily solved by postulating that cultural evolution works in the same way genetic evolution does. Of course, memeticists are aware of the peculiarities of cultural transmission with respect to genetic inheritance (see Sect. 2 above), as well as of the different paces of cultural evolution and genetic evolution. Nevertheless, these are aspects that empirical research is called to elucidate: they do not necessarily invalidate the analogy (as a matter of fact, even within genetic evolution there are many exceptions to the classical Mendelian and gradualist forms of evolution; see, for instance, Schwartz, this volume).

Naturally, as frequently happens, this apparently easy solution has a high price to pay. Although, in fact, it is true that the analogy between genes and memes may be fruitful even in case it is not perfect (no analogy is!), the burden of proof that the gene is a good model to represent the evolving entities in cultural evolution is on memeticists' side. Memeticists must show that the putative dynamics of memes' replication is consistent with our knowledge about how cultural information is transmitted, and they should ideally provide some evidence that memes indeed exist and are causally efficient. Concerning both issues, however, many difficulties have been raised. I shall summarise here just some of them.

First of all, memeticists are not clear about what entities would exactly count as memes. Memes are arguably not identical to cultural variants (beliefs, skills, traditions, artefacts, etc.), which are commonly considered as a class of phenotypic traits (or, in the case of artefacts, objects carrying information potentially influencing phenotypic traits; Odling-Smee et al. 2003). Since memes are by definition analogous to genes, they should be rather considered as the entities which are responsible for the expression of cultural variants (just like genes are the entities which are responsible for the expression of non-cultural phenotypic traits). However, this leads to a highly speculative picture of the processes of cultural transmission. Differently from what occurs in genetic inheritance, it would seem that in cultural transmission the phenotypic traits are directly transmitted (Sperber 2001; Lewens 2013). In what sense, when we learn a new skill or habit, is there a transmission of something else underlying the skill or the habit? And what about the 
developmental processes supposedly involved in the phenotypic expression of the "memetype"?

The analogy with genes, furthermore, commits memeticists to ascribe to memes a certain fidelity of transmission. Again, it is perhaps not necessary, for the analogy to be tenable, that memetic transmission is as faithful as genetic inheritance. Yet, to the extent that memetic transmission is a process of replication, it may well be expected that the socially learned information is approximatively the same as the one that is transmitted. However, as observed by a number of authors (Sperber 2001 is probably the most wellknown; see also Sterelny 2017), social learning of skills, knowledge and traditions is not a process of copy-paste, but a far more complex process in which information is reformulated according to the subject's expectations and goals. As evidence of this, notice that it is rarely possible to identify welldefined lineages of cultural items or traditions (Wimsatt 1999; Lewens 2013; Morin 2016). Making exception of some rare cases, most interesting cultural traits display a relatively high degree of idiosyncratic variation, which turns the memetic hypothesis quite unlikely.

All these criticisms might perhaps be less pressing if memeticists had been able to provide some empirical evidence of the existence of memes or, at least, of their explanatory import. Neither in this sense, though, memetics seem to have made many steps forward. As some former supporters of memetics themselves noticed (Edmonds 2002, 2005), memetics has failed to produce substantive results. At most, it can be interpreted as a metaphorical (and partial) reformulation of something that can be more fruitfully accounted for within more traditional frameworks of cultural change (Kronfeldner 2011).

\subsection{2.}

\section{The Selectionist Interpretation}

According to the "orthodox" view in dual-inheritance theory, the processes of cultural change and accumulation can be considered as analogous to biological evolution even though cultural variants are not analogous to genes (Richerson and Boyd 2005). As already mentioned in the introduction, for the supporters of the selectionist view, all a process needs in order to be properly treated as an evolutionary process is to exhibit variation, heritability and variation in fitness. A process satisfying these requirements is, in addition, not just an evolutionary process, but a Darwinian one (Lewontin 1970; Huneman 2015).

Before discussing in some detail the selectionist stance concerning the problem of dynamical sufficiency, it is important to avoid some possible misunderstandings. First of all, we have to distinguish the selectionist view from what we may call a "panadaptationist" view of cultural evolution. 
According to a supporter of the selectionist view, cultural evolution might be a kind of Darwinian evolution even when selection was not the only factor of change. As a matter of fact, dual-inheritance theorists envisage, as possible causes of cultural change, a broad set of factors, like drift, migration, directed mutation, etc. (Mesoudi 2011; Baravalle 2019), selection thus being just one of them. What characterises the selectionist view against other interpretations of cultural evolution is, rather, a certain emphasis on the population-level properties of the processes of cultural change.

As we have seen in Sect. 2, individuals in a society transmit cultural information through (biased) social learning. Beyond the idiosyncrasies of each interaction between the members of the population, it is possible to find population patterns of distribution of cultural variants. These patterns reflect the overall action of the interactions between individuals in a way that is suitably conceptualised through an evolutionary framework. As a matter of fact, a society may be profitably conceived as a set of different cultural variants (variation), changing in composition through time, for reasons due (except when other factors like drift, migration, etc. are in act) to the differential capacity of cultural variants to be adopted-in virtue of their usefulness, attractiveness or memorability (variation in fitness).

Of course, this explanation can be interpreted as a merely statistical one. It may be said, for instance, that the population-level description of human societies in terms of changing distributions of cultural variants is nothing more than a useful abstraction (see below, Sect. 3.3). Nonetheless, selectionists seem to have a causally stronger interpretation of the evolutionary framework. In other words, they are committed to the claim that the cultural evolutionary framework is dynamically sufficient. But what does it provide the material support of cultural evolution? In a word, heritability. The repeated social interactions between the members of a population constitute a full-fledged channel of inheritance, alternative to the genetic one, and it is precisely the persistence of this inheritance channel what guarantees dynamical sufficiency to the cultural evolutionary process.

We have already discussed the characteristics of social learning as channel of inheritance in Sect. 2. The point here is that the selectionist interpretation, differently from the replicator interpretation, dismisses the analogy between cultural variants and genes, but still claims that cultural transmission is faithful enough to be fruitfully compared to genetic inheritance. In spite of the many idiosyncrasies of any single episode of transmission of cultural information, selectionists argue that there is something (i.e. the cultural variant) which is preserved (Henrich and Boyd 2002) and this provides a sufficient basis to consider the processes of cultural change as evolutionary and, more precisely, as Darwinian. 


\section{The Populational Interpretation}

By analysing a famous model in cultural evolutionary theory on the effects of conformism in the diffusion of a cultural trait (Henrich and Boyd 1998), Lewens (2015) states thatit

aim $[\mathrm{s}]$ to show that individuals who learn in a conformist manner are likely to do better than individuals who instead rely on learning from their environment, or who learn by imitating a randomly chosen member of the population. [Henrich and Boyd's] model takes account of the effects of learning, but not in a way that relies on a notion of Darwinian struggle, or selection, going on among cultural traits (Lewens 2015, p. 38).

After having extended similar considerations to another set of models (Henrich and Boyd 2002; on the characteristics of cultural transmission), Lewens concludes that "the explanatory pay-off of the evolutionary stance derives from the surprising nature of aggregation, made visible by the use of mathematical models. In neither case does the pay-off derive from a notion of cultural selection" (Lewens 2015, p. 38).

If the notion of cultural selection is not crucial to cultural evolutionary explanations, it is natural to wonder: in what sense are they Darwinian? In order to answer this question, Lewens invokes bibliographic evidence, mainly drawn from Boyd and Richerson' writings and, in particular, Richerson and Boyd (2005). He observes that:

in the opening pages of that book, where their basic methodological and explanatory assumptions are laid out, they [Richerson and Boyd] affiliate their approach to a Darwinian tradition in a manner that does not place selection in the foreground, but which instead stresses the importance of population thinking (Lewens 2015, p. 16; emphasis in the original).

Now, in a frequently cited passage, Mayr states that population thinking is the claim that

All organisms and organic phenomena are composed by unique features and can be described collectively only in statistical terms. Individuals, or any kind of organic entities, form populations of which we can determine the arithmetic mean and the statistics of variation. Averages are mere statistical abstractions; only the individuals of which the populations are composed have reality (1959 [2006], p. 326).

If we adapt this conception to cultural evolution, we may reasonably thinkLewens suggests - that, according to cultural evolutionary theory, only the individual organisms, endowed by specific psychological biases, are "real", while the properties of the populations, like the distribution of cultural variants, are mere statistical abstractions (Lewens 2009, p. 248-9). Cultural change, accordingly, would be-like a change in temperature or pressure in the kinetic theory of gases - just the aggregate of myriads of individual-level interactions: no population-level factors would play a causal role in addition to these interactions. Population-level descriptions certainly have an 
invaluable explanatory import, because they allow to bookkeep the otherwise epistemically inaccessible contingent circumstances in which individual organisms learn and transmit information. Nevertheless, the population-level properties that occur in such descriptions are causally inert, mere epiphenomena, which are in need to be further explained in terms of individual-level interactions.

This interpretation is also shared by a group of cultural evolutionists frequently labelled as cultural epidemiologists (Sperber 1996; Claidière et al. 2014; Morin 2016; Scott-Phillips et al. 2018). Besides being critics of memetics, they are sceptical about the selectionist interpretation for similar reasons: in their opinion, cultural transmission is not faithful enough to be properly considered as a channel. During the many interactions between individuals in a population, cultural information is continuously reformulated. While, a posteriori, it is frequently possible to reconstruct a unitary narrative of certain cultural traditions, there is nothing like $a$ cultural trait persisting through all the interactions. This may be interpreted as a claim that, as evolutionary theory, cultural evolutionary theory is not dynamically sufficient. Of course, there is "something underlying" the process of cultural change, but cultural change in itself is not a phenomenon admitting an autonomous causal characterisation (contrariwise genetic evolutionary processes).

\subsection{4.}

\section{Provisional Remarks}

As already mentioned in the introduction, my goal in this chapter is to show that there is at least another alternative way to interpret a process as evolutionary and, more specifically, as Darwinian-besides the ones usually considered in the literature. Before proceeding to that, however, I would like to pause our discussion and highlight some, in my opinion, important points. First of all, I would like to stress that the interpretation of population thinking advocated by Lewens and cultural epidemiologists is just one among others possible. I argued more extensively for this claim elsewhere (Baravalle 2019), but I think it is useful here to shortly return on it. According to two champions of dual-inheritance theory, McElreath and Henrich (2007), for instance, population thinking does not entail a reduction of populationlevel dynamics to individual-level interactions (this would be more characteristic of methodological individualism in social sciences) nor a reduction of individual-level interactions to population-level dynamics (this would be methodologically equivalent to adopting a sort of holism). Instead, it entails an interplay between the two levels. Given a certain demic structure, individuals interact with neighbours and with the environment, thus determining the composition of the population in terms of cultural variants. 
However, the other way around, cultural variants influence, in virtue of their distribution and functional properties, the demic structure and, therefore, individuals' behaviours and interactions. A similar conception was indeed already defended by Boyd and Richerson (1985, pp. 23-4).

According to this alternative interpretation of population thinking - which is in contrast with the one supported by Lewens and cultural epidemiologistsin order to explain cultural change it is not enough to decompose the phenomenon into its basic elements, but it is also necessary to take into account the characteristics of the population as a whole. To provide a complete picture of cultural change, populations must be represented as "changing distributions of cultural variants". This is not simply a useful description of something that can also be expressed otherwise (i.e. in terms of additive individual interactions); it denotes a standalone feature of the processes being modelled.

Even admitting this alternative view of population thinking, which is certainly more friendly towards a selectionist interpretation of cultural evolutionary processes, the problem of the fidelity of cultural transmission is still there. On the one hand, and independently from any specific conception of population thinking, a purely populational interpretation of cultural change, although perhaps sufficient to characterise cultural evolutionary theory as "evolutionary" (in the sense that it provides information about the entities carrying the causal weight of the processes under study), is probably too weak to make it a "Darwinian" theory. Not just Darwin's theory, but many other theories which we would not consider as properly Darwinian-for instance, in epidemiology, sociology or economics - adopt some populational view akin to the ones presented in this and the last subsection (see Illari and Russo 2014, Chap. 5).

On the other hand, the issue is that - if we consider the selectionist view as the one stating the minimal requirements for a theory to be Darwinian-it is not clear that cultural transmission is faithful enough to count as an inheritance channel. I think that this problem is prominently empirical. In this sense, further research on the nature of the transmission of information in social interactions and the social learning processes is certainly needed (and it is already ongoing; see, for instance, Kendal et al. 2018). In the remainder of this chapter, though, I shall suggest that all this debate is somehow vitiated by an unwarranted assumption, that is, that the dynamical sufficiency of cultural evolutionary theory entirely (or, at least, mostly) depends on the fidelity of cultural transmission.

\section{4}




\section{Demographic Factors in Genetic and Cultural Evolution}

In Sect. 2, we have seen that the theory of genetic evolution is a full-fledged evolutionary theory, in conformity with Lewontin's characterisation, because it supposedly provides a set of transformation laws and a dynamical characterisation of the processes under study. This theory is dynamically sufficient because it identifies the factors that are materially responsible for the transformations in the state space of allelic frequencies: these factors are genetic inheritance and the differential survival and reproduction of the individuals due to environmental circumstances. The debate over the evolutionary interpretation of cultural evolutionary theory discussed in Sect. 3 has been almost entirely focused on the putative similarities between cultural transmission and genetic inheritance, largely neglecting the importance of environmental factors in the dynamical characterisation of evolutionary processes. In this section, I shall focus on a type of environmental factors that, in my opinion, are especially relevant in the causal characterisation of cultural evolution, that is, demographic factors.

Interestingly, demographic factors have been somewhat neglected also in evolutionary biology. For this reason, I shall first, in Sect. 4.1, briefly discuss how they have been recently incorporated within traditional models of genetic evolution. Then, in Sect. 4.2, I shall argue that they have always played a prominent role also in cultural evolutionary models. In Sect. 5, I shall finally show how the consideration of demographic factors helps to provide a more articulated Darwinian picture of cultural evolutionary theory.

\subsection{1.}

\section{Demographic Factors in Genetic Evolution}

Although, as we shall discuss in some detail in Sect. 5, the relevance of demographic factors in evolution was already stressed by an architect of the Modern Synthesis such as Sewall Wright, it is just in the last 30 years or so that their importance in bridging ecological and evolutionary knowledge has started to be fully appreciated. As Lowe et al. (2017) notice:

The rift between longstanding population genetics theory and current ecoevolutionary research underscores the challenge of fully addressing the forces that drive evolution at the population level. This challenge is not new ([Darwin 1859], p. 64), and is embodied in a historical debate over the power of selection to drive evolutionary change in the face of other, non-adaptive forces-a debate that is largely settled in the field of evolutionary biology, but the root of a narrow view of evolution in many current eco-evolutionary studies (Lowe et al. 2017, p. 142).

In Lowe and his colleagues' opinion, the study of the demic structure of a population is the key to understand the conditions under which selective 
pressures and other evolutionary forces can act, and with which intensity. Lowe and colleagues identify three main properties of a population that can bias evolutionary dynamics: population size, population density and population connectivity.

Population size is, perhaps, the less surprising one. As a matter of fact, the effects of population size on population dynamics has long been noticed, both theoretically (Wright 1932) and experimentally (Dobzhansky and Pavlovsky 1957). Typically, a reduced population size prevents natural selection to act sorting the fittest traits, increases stochastic fluctuations of allelic frequencies (i.e. genetic drift) and, ultimately, results in a reduction of variation. The other way around, to the extent population size (or, more correctly, effective population size; Charlesworth 2009) increases, drift effects diminish and natural selection can act undisturbed.

The effects of population density are more interesting. Population density favours processes of soft selection (Wallace 1975). In Lowe et al.'s words:

Under soft selection, the availability of different niches in the environment and competition among genotypes results in adaptive change. There are no optimal genotypes, but instead a range of successful genotypes determined by the combination of extrinsic forces, density and the frequency of other genotypes in the population (Lowe et al. 2017, pp. 142-143; emphasis added).

In other words, soft selection-which, contrarily to "hard" selection, is not an eliminative force, but permits the maintenance of a broad range of different phenotypes - is a force directly related to demographic factors. We may say that population density favours specialisation. The high density of a population is generally the effect of the absence of ecological factors causing hard selection (like, for instance, predators or the extreme scarcity of resources, which yield a high mortality). In such conditions, as Darwin had already noted, the "struggle for survival" may acquire less dramatic tones; nonetheless, since resources are usually not unlimited, selection can act more subtly, by promoting survival strategies that resort to resources not exploited by other members of the population. ${ }^{4}$

Finally, population connectivity is surely the most complex and heterogenous demographic cause of evolution. As a matter of fact, it would be more correct to conceive population connectivity as a class of demographic factors, rather than a single one. What all these factors have in common is that they depend on migration between demes in a structured population. The migration of individuals from a deme to another may generate a series of evolutionary dynamics, from the promotion or the prevention of adaptive evolution, to stochastic fluctuations. Notably, one of the contexts in which the effects of migration in a connected population have been most extensively studied is in the diffusion of behavioural traits. Animal personality, for instance, has been linked to different eco-evo dynamics (Sih et al. 2012). Animals 
with "exploratory" personalities, who are more propense to migrate than "shy" ones, can be heavily influential in the behaviour of the individuals belonging to the deme which receives them. Populations with a high level of connectivity are more subject to this kind of "invasions" than less connected ones, and different demic structures may favour or prevent a broad range of possible dynamics.

A common feature of all these demographic factors is that they can act very quickly, from an evolutionary point of view, reflecting in almost real-time the effects of the ecological circumstances in which the population happens to find itself. The demographic factors accelerate complex evolutionary dynamics affecting the composition of the population, even when the traits undergoing the evolutionary dynamics have not a well-defined genetic basis (or it is unknown).

\subsection{2.}

\section{Demographic Factors in Cultural Evolution}

All the demographic factors discussed in the previous sub-section have also been studied in the context of cultural evolution.

Concerning the effects of population size, the possibly most well-known case is the loss of technology in Tasmania, originally described in cultural evolutionary terms by Henrich (2004). About 18,000 years ago, New Guinea and Tasmania were a unique land with Australia. When the process of insulation started, Tasmanian populations found themselves separated from their Australian cousins. This resulted in a drastic reduction of the population size of Tasmanian populations. One of the effects of this geological transformation was a quick loss of technologies, especially related to fishing, in Tasmanian populations. Like in genetic evolution, also in cultural evolution population size reduction entails a reduction of variation in the population (in the Tasmanian case, the death of fishermen who never taught fishing techniques irremediably resulted in the disappearance of such techniques).

Population density and connectivity are both decisive factors, according to Lycett and Norton (2010), for the emergence of Palaeolithic technological evolution. In their opinion, we can reconstruct three ideal stages of such evolution (occurred during different epochs depending on the geographical region), roughly corresponding to three distinct "demographic levels", or modes. In the first level, human populations are relatively small, with low density and weak interconnectedness. Human populations at this stage exhibit little technological variation. The second and the third modes correspond to a progressive enlargement of human populations, a greater density and higher levels of social interconnectedness. All this contributes to a more diverse cultural variation (similar conclusions are attained by Powell et al. 2009). 
These studies do not reveal what kind of cultural variants are favoured by population density and connectivity, but just that these are crucial conditions for having cultural variation. Nonetheless, other works developed in the context of cliodynamics (Turchin 2003, 2008) seem to provide more information about frequency-dependent dynamics between specific kinds of cultural variants throughout the history of human societies. Cliodynamics is a dynamical systems approach applied to human history, which accounts for the evolution of a society as the effect of the interaction between many subsystems. In order to formulate reliable hypotheses about historical phenomena, Turchin and his collaborators elaborate data from Seshat, an impressive databank which collects information about the social and political organisation of virtually any human group along the history of our species, from Neolithic to modern societies (http://seshatdatabank.info).

Turchin et al. (2018) provide surprising evidence about the predictability of many characteristics of complex societies across different world regions. These researchers captured information on 51 variables reflecting nine characteristics of human societies, such as social scale, economy, features of governance and information systems. Even though Cliodynamics adopts an approach which is different from the one adopted by dual-inheritance theory, we may reasonably consider such variables as classes of cultural variants. Turchin et al. found out that some aspects of social organisation are functionally connected and, for this reason, the classes of behaviour related to them are expected to coevolve in a predictable way. For instance, scale variables are likely to be tightly linked, since increases or decreases in size may require changes in the degree of hierarchy (both too few and too many decision-making levels create organizational problems). A similar argument has been put forward for size and governance. The production of public goods, such as infrastructure, may require solutions to collective action problems, and these can be provided by governance institutions and professional officials (Turchin et al. 2018, p. E147).

The spread of religious, philosophical or scientific beliefs are, as it may be expected, correlated with the diffusion of information systems, such as alphabet and writing. On the contrary, other classes of variables, like those related to money, are just loosely related to the evolution of most other variants. However, all these variables are somehow dependent on the demographic and organisational features of the societies.

\section{5 .}

\section{Is this Darwinism?}

All the evidence discussed in the previous section supports, in my view, the claim that, even if we eventually discovered that cultural transmission is 
significantly different from genetic inheritance, cultural evolutionary theory would keep being to a certain extent dynamically sufficient in virtue of demographic factors. Demographic factors seemingly play a crucial role both in the emergence and in the evolution of culture. They are what Wimsatt (2019) would call an "external scaffold" of cultural evolution: a set of structural features that are, at the same time, the condition of possibility of cultural dynamics, and determine (although, of course, with a certain flexibility) the paths that societies are likely to follow once certain cultural variants spread.

We are now ready to reconsider our original question: is a, so to speak, "evodemo" theory of cultural evolution Darwinian? The question is interesting because it has long been thought that the notions of population involved, respectively, in demographic and evolutionary studies were different and, to a large extent, incompatible (Kreager 2009). While Darwinian populations would be characterised by the variation between the members that make them up (in accordance with the interpretation of population thinking supported by Lewens and cultural epidemiologists), the notion of population at stake in demography_especially after the work of Alfred Lotka (1925)—would stress "typological" features of populations (such as fertility, mortality, etc.), shared by all of them. The two notions of population also differ because they suggest distinct conceptualisations of the environment in which a population is found. On the one hand, Darwinian populations are typically conceived as "open" populations, in the sense that they are sensitive to ecological influences, which can constitute selective pressure and, thus, change the composition of the population. Lotkean populations, on the other hand, would be "closed", in the sense that the demographic factors are intended as sufficient for determining the changes that the population undergo through time.

These differences between Darwinian and Lotkean populations-although perhaps methodologically relevant in some practices of, respectively, evolutionary biologists and demographers - are not, as far as I can see, an impediment to a fruitful synthesis between the two approaches. As a matter of fact, the basis for this synthesis was already laid, long time ago, by a fervent Darwinist, that is, Sewall Wright.

The work of Wright is best understood in opposition to that of another Darwinian, Ronald Aylmer Fisher. Fisher first exposed his theoretical proposal about evolutionary processes in a series of papers between the 1910s and 1920s and later, more in detail, in The Genetical Theory of Natural Selection (1930). Fisher's theory of evolution has been traditionally considered as a synthesis between the hitherto incompatible views of biometricians and Mendelians. In Fisher's view, Biometricians were right in believing that phenotypic variation is continuous and that this fact requires an explanation, but they erroneously explained the transgenerational change 
in the frequencies of phenotypic variants by postulating a mechanism of blending inheritance and massive mutations. On the other hand, Mendelians correctly believed that the units of inheritance - that is, of course, geneswere discrete entities and that Mendelian inheritance was sufficient to guarantee the large amount of variability necessary to undertake the evolutionary processes, without postulating massive mutations. Nevertheless, they were wrong in underestimating the creative power of natural selection. 5 The challenge was thus, for Fisher, to explain how discrete entities like genes could generate continuous phenotypic variation and gradual evolutionary novelties. To this goal, he conceived allelic genes as individually responsible for very small phenotypic differences and selectable for any, even weak, adaptive benefit. Fisher conceived natural populations, at a certain generation, as arrays of alleles distributed along a multidimensional Gaussian curve, with the most common combinations in the middle. Ideally, an indefinitely large panmictic population where no evolutionary force is acting persists in this equilibrium. However, this is not what happens in the real world: in real populations, this equilibrium is constantly perturbed. Fisher considered that natural selection is the single most relevant cause of adaptive change in genetic frequencies. It constantly operates, in Fisher's view, on every single locus - with completely addictive effect - by eliminating less fit genotypes, thus gradually increasing the average fitness of the population. This claim is expressed by Fisher's Fundamental Theorem of Natural Selection, according to which "the rate of increase in the mean fitness of a population ascribable to gene-frequency changes is exactly equal to the additive genetic variance in fitness" (Edwards 2002, p. 335).

Wright regarded at Fisher's model as overly simplistic (Wright 1931). Following a typical Darwinian modus operandi (that is, the comparison between natural and artificial populations), he observed that when breeders try to select a single advantageous character in a large population (this technique is usually called "mass selection"), the consequences are frequently negative: "mass selection has a tendency to lower fitness in the population by turning up all sorts of unwanted gene combinations, normally hidden in heterozygotes, eventually inducing infertility" (Depew and Weber 1996, p. 280). Even when this is not the case, the fixation of the selected trait is extremely slow. In Wright's opinion, the effectiveness of mass selection is severely limited by the fact that genes usually do not phenotypically express themselves separately, but through complex networks of interactions, which are not necessarily genetically inheritable. A more effective way to select a trait would be to breed only those specimens whose networks of interactions are genetically transmissible as a whole.

Unfortunately, it is difficult to identify such specimens. In order to overcome this difficulty, "skilled breeders take the best specimens that can be produced by inbreeding and then outbreeding them with the best specimens of a 
separate population" (Depew and Weber 1996, p. 280). The inbreeding phase favours the expression of a variety of inheritable interaction networks, thus allowing selection to act more efficiently. Wright guessed that something similar had to happen in nature as well. Consequently, he conceived the threestep model known as shifting-balance model. According to it, species in nature are subdivided in relatively small inbreeding sub-populations, called demes. During a first phase, every deme, due to its reduced size, undergoes genetic drift. This process favours genetic recombination and increases selectable variation. Once inheritable interaction networks are made phenotypically visible, mass selection may act on the fittest variants, eventually raising the mean fitness of some demes (second step). Finally, interdemic selection, favoured by the interdemic migration of the most adapted individuals, raises global fitness (third step). In order to represent his model graphically, Wright conceived the famous diagram known as adaptive landscape (Wright 1932), depicting the evolutionary space as a complex array of valleys and hills, instead of a linear pathway to adaptation, as in Fisher's conception.

Through his shifting-balance model, Wright overcame a simplistic distinction between open and closed populations. Demes within a metapopulation, as well as the metapopulation itself, are open and closed at the same time, and both "openness" and "closedness" play a crucial role in population dynamics. It is precisely because demes are closed that they can undertake those processes (i.e. drift and recombination) that eventually permit intrademic selection to act. At the same time, demes need to be open to allow migration and interdemic selection to act. At a greater scale, the metapopulation is open insofar as, as a whole, it is subject to environmental conditions that influence its composition. Yet, in another sense, it is closed because its demic structure is a "typological" condition, which is necessary in order to produce the characteristic outcome of the shifting-balance process.

Nowadays, most biologists consider that the shifting-balance model is, strictly speaking, flawed (Coyne et al. 1997). This is because the three phases of the shifting-balance process predicted by Wright have been rarely (if ever) observed. Nonetheless, this does not diminish the importance of Wright's approach. Even though natural populations do not behave exactly like Wright thought, the consideration of the structural and demographic features of populations is, in many scenarios, crucial for correctly depicting evolutionary processes (Wade and Goodnight 1998; Wade 2016; see also Sect. 4.1 above). All this reinforces the impression that, in the context of cultural evolution, the debate about "how Darwinian" is cultural evolutionary theory has been artificially narrowed. First of all, it is not necessary that natural selection is ubiquitous for considering an evolutionary dynamic as Darwinian (see Bellon, Delisle; this volume). In Wrightian dynamics, selection is always accompanied by many other processes modulating the population change. 
This is indeed something that the supporters of the selectionist approach acknowledge, but it has not been fully appreciated by Lewens and cultural epidemiologists in their criticisms. Both the selectionist and the populational interpretations, nonetheless, are limited to the extent that they focus almost exclusively on the putative resemblances (or differences) between genetic and cultural inheritance. The persistence of a relatively faithful channel of inheritance is, of course, a fundamental condition for the implementation of a Darwinian process. Nevertheless, there are other factors that can play an important role in directing evolutionary dynamics.

As seen in Sect. 4, demographic factors are powerful causes of evolution. They sometimes simply favour some specific kinds of selective dynamics. However, in other cases, they create the conditions for more complex dynamics to occur. The demographic features of a population can drive the population towards certain specific compositions, in terms of cultural variants, or even to large-scale transitions (like in the case of Palaeolithic technological evolution).

\section{6.}

\section{Conclusion}

In this chapter, I have assessed some explanatory and methodological issues related to the possibility of considering the theory of cultural evolution as a Darwinian theory. First of all, in Sect. 2, I have presented a minimal characterisation of an evolutionary theory as a theory containing a set of transition laws and a dynamical characterisation of those laws (i.e. a causal description of the evolving system). The genetic theory of evolution is an evolutionary theory because it provides a set of transition laws describing the change of the allelic frequencies in a population, and it dynamically characterises this process by spelling out the mechanisms governing genetic inheritance and the possible ecological factors instantiating evolutionary forces.

In the context of cultural evolutionary theory, there is a disagreement concerning whether it is possible to provide a causal characterisation of cultural change that is, to some extent, analogous to that of the genetic theory of evolution. In this debate, it is more or less implicitly assumed that it is depending on the similarities between genetic inheritance and cultural transmission that it is possible to qualify cultural evolutionary theory as a Darwinian theory (and cultural change as a Darwinian process). In Sect. 3, I have reviewed the three main positions in this debate, and I have concluded that the plausibility of two of them-that is, the selectionist and the populational interpretations - has to be assessed in light of empirical evidence. Still, I have also argued that the emphasis on the similarities or the 
differences between genetic inheritance and cultural transmission has obscured other possible similarities between biological and cultural evolution.

In Sect. 4, have stressed that demographic factors-understood as a set of factors related to the structure of populations - are calling attention of both evolutionary biologists and cultural evolutionists as an important cause of evolution. The point relevant for the debate concerning cultural evolution is that, even if we found out that cultural transmission is dissimilar to genetic inheritance in crucial aspects, this would not imply that we need to give up the idea that cultural evolutionary theory is dynamically sufficient in a way that resembles evolutionary theory in biology. To address the concern that such a theory, although evolutionary, would not be Darwinian, I have argued in Sect. 5, that there is a long tradition in evolutionary biology that considers demographic factors as influential in evolutionary dynamics. This way of conceptualising evolutionary change, albeit perhaps not strictly speaking Darwinian, would be, at least, Wrigthean .

In conclusion, regarding the role of selection in cultural evolution and the appropriateness of considering cultural change as a selective process, I would say that this should not be the focus of the discussion. Indirectly, the same may be said concerning the broader debate about the centrality of natural selection in biological evolutionary theory. Natural selection is certainly a fundamental process in evolution, and a fundamental process in the original Darwinian formulation of his theory. Nonetheless, theories change through time. In the case of biological evolutionary theory, the Modern Synthesis and, especially, the work of Sewall Wright showed that Darwinian theory is able to incorporate elements that were not included in the Darwinian formulation of the theory (as also emphasised by Esposito, this volume). Rather than discussing whether cultural evolutionary theory is a Darwinian theory because it properly mimics certain aspects of the genetic theory of evolution, we should be open to extend our analysis to other-perhaps sometimes considered as peripheral-aspects of evolutionary biology.

In this sense, the parallel drawn in Sects. 4 and 5 between genetic and cultural evolution concerning the impact of demographic factors in evolution should not be just considered as an invitation to cultural evolutionists to seek the Darwinian origins of cultural evolutionary theory outside the debate over the similarities between genetic inheritance and cultural transmission. Also, it has the goal of stressing the ability of Darwinian theory of including topics and descriptions of evolutionary phenomena elaborated within other disciplinary fields, even when these descriptions do not put selection in the foreground.

\section{Acknowledgements}


I would like to thank the Brazilian National Council for Scientific and Technological Development (CNPq Grant $\left.N^{\circ} 402619 / 2016-1\right)$ and the Fundação para a Ciência e a Tecnologia de Portugal (FCT Contract $\mathrm{N}^{\circ}$ DL57/2016/CP1479/CT0064) for the financial support.

\section{References}

Aguilar E, Akçay E (2018) Gene-culture co-inheritance of a behavioral trait. Am Nat 23:311-320

Aunger R (2001) Introduction. In: Aunger R (ed) Darwinizing culture: the status of Memetics as a science. Oxford University Press, Oxford, pp 1-23 Baravalle L (2019) Cultural evolutionary theory as a theory of forces. Synthese. https://doi.org/10.1007/s11229-019-02247-0

Blackmore S (1999) The meme machine. Oxford University Press, Oxford Boyd R, Richerson PJ (1985) Culture and the evolutionary process. The University of Chicago Press, Chicago

Brusse C (2017) Making do without selection. Biol Philos 32:307-319

Cavalli-Sforza L, Feldman M (1981) Cultural transmission and evolution: a quantitative approach. Princeton University Press, Princeton

Charlesworth B (2009) Effective population size and patterns of molecular evolution and variation. Nat Rev Genet 10:195-205

Claidière N, Scott-Phillips TC, Sperber D (2014) How Darwinian is cultural evolution? Philos Trans R Soc Lond Ser B Biol Sci 369

Coyne JA, Barton NH, Turelli M (1997) Perspective: a critique of Sewall Wright's shifting balance theory of evolution. Evolution 51:643-671

Darwin C (1859) On the origin of species by means of natural selection. Murray, London

Dawkins R (1976) The selfish gene. Oxford University Press, Oxford Dennett DC (1995) Darwin's dangerous idea: evolution and the meaning of life. Simon \& Schuster, New York

Dennett DC (2017) From bacteria to Bach and back: the evolution of minds. W. W. Norton, New York

Depew DJ, Weber BH (1996) Darwinism evolving: system dynamics and the genealogy of natural selection. The MIT Press, Cambridge (MA)

Dobzhansky T, Pavlovsky O (1957) An experimental study of interaction between genetic drift and natural selection. Evolution 11:311-319

Durham WH (1991) Coevolution: genes, culture, and human diversity. Stanford University Press, Stanford

Edmonds B (2002) Three challenges for the survival of memetics. JOM 6

Edmonds B (2005) The revealed poverty of the gene-meme analogy - why memetics per se has failed to produce substantive results. JOM 9

Edwards AWF (2002) The fundamental theorem of natural selection. Theor Popul Biol 61:335-337 
El Mouden C, André J-B, Morin O, Nettle D (2014) Cultural transmission and the evolution of human behaviour: a general approach based on the Price equation. J Evol Biol 27:231-241

Fisher RA (1930) The genetical theory of natural selection. Clarendon, Oxford

Godfrey-Smith P (2007) Conditions for evolution by natural selection. J Philos 104:489-516

Godfrey-Smith P (2009) Darwinian populations and natural selection. Oxford University Press, Oxford

Gould SJ (1977) Ontogeny and phylogeny. Harvard University Press, Cambridge (MA)

Henrich J (2001) Cultural transmission and the diffusion of innovations: adoption dynamics indicate that biased cultural transmission is the predominate force in behavioural change. Am Anthropol 103:992-1013

Henrich J (2004) Demography and cultural evolution: how adaptive cultural processes can produce maladaptive losses: the Tasmanian case. Am Antiq 68:197-214

Henrich J (2016) The secret of our success: how culture is driving human evolution domesticating our species and making us smarter. Princeton University Press, Princeton (NJ)

Henrich J, Boyd R (1998) The evolution of conformist transmission and the emergence of between-group differences. Evol Hum Behav 19:215-242

Henrich J, Boyd R (2002) On modeling cultural evolution: why replicators are not necessary for cultural evolution. J Cogn Cult 2:87-112

Huneman P (2015) Selection. In: Heams T, Huneman P, Lecointre G, Silberstein M (eds) Handbook of evolutionary thinking in the sciences. Springer, Dordrecht, pp 37-76

Illari P, Russo F (2014) Causality. Philosophical theory meets scientific practice. Oxford University Press, Oxford

Kendal RL et al (2018) Social learning strategies: Bridge-building between fields. Trends Cogn Sci 22:P651-P665

Kimura M (1968) Evolutionary rate at the molecular level. Nature 217:624626

King JL, Jukes TH (1969) Non-Darwinian evolution. Science 3881:788-798 Kreager P (2009) Darwin and Lotka: two concepts of population. Demogr Res 21:469-502

Kronfeldner M (2011) Darwinian creativity and memetics. Routledge, New York

Lewens T (2009) Innovation and population. In: Krohs U, Kroes P (eds) Functions in biological and artificial worlds: comparative philosophical perspectives. The MIT Press, Cambridge (MA), pp 243-258

Lewens T (2013) Cultural evolution. In: ZaltaEN (ed), The Stanford encyclopedia

of philosophy,

Spring 2013 
edition. http://plato.stanford.edu/archives/spr2013/entries/evolutioncultural/

Lewens T (2015) Cultural evolution. Oxford University Press, Oxford Lewontin RC (1970) The units of selection. Annu Rev Ecol Syst 1:1-18

Lewtonin RC (1974) The genetic basis of evolutionary change. Columbia University Press, New York

Lotka AJ (1925) Elements of physical biology. Williams \& Wilkins, Baltimore

Lowe WH, Kovach RP, Allendorf FW (2017) Population genetics and demography unite ecology and evolution. Trends Ecol Evol 32:141-152

Lycett SJ, Norton CJ (2010) A demographic model for Palaeolithic technological evolution: the case of East Asia and the Movius Line. Quatern Int 211:55-65

Matthen M, Ariew A (2002) Two ways of thinking about fitness and natural selection. J Philos 99:55-83

Mayr E (1959 [2006]) Typological versus population thinking. In: Sober E (ed) Conceptual issues in evolutionary biology. The MIT Press, Cambridge (MA), pp 325-328

McElreath J, Henrich G (2007) Modelling cultural evolution. In: Dunbar R, Barrett L (eds) Oxford handbook of evolutionary psychology. Oxford University Press, Oxford, pp 571-585

Mesoudi A (2011) Cultural evolution: how Darwinian theory can explain human culture and synthesize the social sciences. The University of Chicago Press, Chicago

Morin O (2016) How traditions live and die. Oxford University Press, Oxford Odling-Smee FJ, Laland KN, Feldman MW (2003) Niche construction: the neglected process in evolution. Princeton University Press, Princeton

Pigliucci M, Muller G (2010) Evolution - the extended synthesis. MIT Press, Cambridge

Powell A, Shennan S, Thomas MG (2009) Late Pleistocene demography and the appearance of modern human behavior. Science 324:1298-1301

Richerson PJ, Boyd R (2005) Not by genes alone: how culture transformed human evolution. The University of Chicago Press, Chicago

Scott-Phillips T, Blancke S, Heintz C (2018) Four misunderstandings about cultural attraction. EvolAnthropol 27:162-173

Sih A et al (2012) Ecological implications of behavioural syndromes. Ecol Lett 15:278-289

Sperber D (1996) Explaining culture. A naturalistic approach. Blackwell, Oxford

Sperber D (2001) An Objection to the memetic approach to culture. In: Aunger R (ed) Darwinizing culture: the status of Memetics as a science. Oxford University Press, Oxford, pp 163-174 
Sperber D, Claidière N (2008) Defining and explaining culture (comments on Richerson and Boyd, Not by genes alone). Biol Philos 23:283-292

Sterelny K (2017) Cultural evolution in California and Paris. Stud Hist Phil Biol Biomed Sci 62:42-50

Turchin P (2003) Complex population dynamics: a theoretical/empirical synthesis. Princeton University Press, Princeton

Turchin P (2008) Arise "Cliodynamics". Nature 454:34-35

Turchin P et al (2018) Quantitative historical analysis uncovers a single dimension of complexity that structures global variation in human social organization. PNAS 115:E144-E151

Wade MJ (2016) Adaptation in metapopulations. University of Chicago Press, Chicago

Wade MJ, Goodnight CJ (1998) Genetics and adaptation in metapopulations: when nature does many small experiments. Evolution 52:1537-1553

Wallace B (1975) Hard and soft selection revisited. Evolution 29:465-473

Wimsatt WC (1999) Genes, memes, and cultural heredity. Biol Philos 14:279-310

Wimsatt WC (2019) Articulating Babel: a conceptual geography for cultural evolution. In: Love AC, Wimsatt WC (eds) Beyond the meme. University of Minnesota Press, Minneapolis, pp 1-41

Wright SW (1931) Evolution in Mendelian populations. Genetics 16:97-159 Wright SW (1932) The roles of mutation, inbreeding, crossbreeding, and selection in evolution. Proceedings of sixth international congress on genetics $1: 356-366$

Wright SW (1948) On the roles of directed and random changes in gene frequency in the genetics of populations. Evolution 2:279-294

Wright SW (1982) The shifting balance theory and macroevolution. Annu Rev Genet 16:1-19

\section{Footnotes}

1 To be precise, Lewontin introduces a third requirement to be satisfied by evolutionary theories, that is, empirical sufficiency. An evolutionary theory is empirically sufficient if scientists are able to measure the parameters that they introduce to formulate the laws of transformation. This is tantamount to say that the causal representation offered by an evolutionary theory should not be attainable just in principle, but also in practice. Lewontin is sceptical about the capacity of the genetic theory of evolution to fully satisfy this requirement. This is certainly a problem also for cultural evolutionary theory, but I shall not discuss it directly here.

$\underline{2}$ Notice that cultural selection does not necessarily favour genetically fittest variants. Since social learning is usually less costly or more effective than individual trial-and-error, partially maladaptive cultural variants (such as certain unhealthy eating habits) may be maintained within a population, evolve and even subvert "genetically-coded" behaviours.

$\underline{3}$ This is arguably the case of "purely statistical" theories (Matthen and Ariew 2002).

${ }^{4}$ This is indeed, according to some authors, the most common scenario in evolution (see, for a discussion of ideas related to this claim, Tattersall's chapter in this volume). 
${ }^{5}$ For some more accurate remarks on the controversy between Mendelians and biometricians, and the origins of population genetics, see Adams's chapter and Ochoa's first contribution in this volume. 